\title{
Los poliminoes: una experiencia geométrica y espacial con estudiantes de cuarto grado de Básica Primaria*
}

\author{
THE POLIMINOES: GEOMETRIC AND SPATIAL EXPERIENCE WITH FOURTH GRADE STUDENTS \\ OF BASIC PRIMARY
}

OS POLIMINOES: UMA EXPERIÊNCIA GEOMÉTRICA E ESPACIAL COM ESTUDANTES DE QUARTO GRAU DE BÁSICA PRIMARIA

Johnson Vicente Bohórquez Torres** / johnsonbohorquez@hotmail.com

\section{Resumen}

Se presenta la sistematización de la construcción de poliminoes con estudiantes de grado cuarto del colegio Gimnasio Colombo Británico. Las estrategias fueron la construcción de la figura en papel, el diseño en el programa Paint y su procedimiento en el Lenguaje Logo. Las intenciones de la actividad fueron desarrollar habilidades de pensamiento geométrico y espacial y experimentar con los comandos del Logo. Se realizaron clasificaciones, análisis y reconocimiento de las características de las figuras, una comprensión de los conceptos de perímetro y área, y la recreación de las construcciones en el ordenador.

\section{Abstract}

We present the systematic poliminos' construction with the fourth grade students of the "Gimnasio Colombo Británico" school. The strategies that were usedwere the construction of the figure on paper, the design of them in the Paint Softwareits procedure in the Logo software. The activity intentions' are to develop skills in geometry and spatial thinking, and experimenting with the Logo commands.In the activity was allowed to perform classifications, analysis and recognition of the figures characteristics' understand theperimeter and area concepts and recreate these constructions on the computer.

\section{Resumo}

Apresentamos a construção sistemática de poliminoes comquartoestudantes da classe do "Gimnásio ColomboBritanico" escola. As estratégiasforam a construção da figura em papel, o desenho no programa Paint e procedimento no idioma Logo. As intenções da atividade foi desenvolver habilidades de pensamento e de geometria espacial, experiência com comandos do logotipo. Classificações foram feitas, a análise e reconhecimento das características das figuras, entender os conceitos de perímetro e área e recriar os prédios no computador.

\section{Palabras clave}

Pensamiento geométrico, pensamiento espacial, poliminoes, figuras, procedimiento.

\section{Keywords}

Geometric thinking, spatial thinking, poliminos, figures, procedures.

\section{Palavras-chave}

Pensamento geométrico, raciocínio espacial, poliminoes, figuras, processo.

\footnotetext{
* El artículo se constituye a partir de las actividades adelantadas en el Seminario de Sistematización en las prácticas de Enseñanza de las Ciencias, de la Maestría en Docencia de las Ciencias Naturales de la Universidad Pedagógica Nacional.

** Licenciado en Física y estudiante de la Maestría en Docencia de las Ciencias Naturales. Departamento de Física de la Universidad Pedagógica Nacional. Profesor del Colegio Gimnasio Británico.
} 


\section{Presentación}

En el artículo se presenta la sistematización de una actividad en el aula con los estudiantes del grado cuarto del Colegio Gimnasio Colombo Británico, Bogotá. La experiencia se concentró en la construcción de poliminoes teniendo en cuenta dos estrategias: la construcción de la figura en papel y su dibujo en el programa Paint, como ejercicios primarios que permiten reconocer e identificar el conocimiento de los estudiantes sobre la figura cuadrado y la comprensión del concepto de perímetro y área, para finalmente construir los poliminoes utilizando el Lenguaje Logo.

En el proceso de sistematización se tuvieron en cuenta dos intenciones: la primera, la relación del trabajo con el Lenguaje Logo, en el cual se contempló experimentar con los comandos de Logo para entenderlos y alcanzar confianza es su uso; esto significó: planear el trabajo para organizar los componentes; escribir un conjunto de instrucciones para realizar cada tarea; evaluar el programa al localizar y corregir errores para comprender el método utilizado y construir un programa para realizar todas las tareas en el orden adecuado. El entorno gráfico posibilita a los estudiantes saber donde están ubicados para iniciar el trabajo, mientras el lenguaje Logo permite la representación visual de conceptos matemáticos y geométricos, de tal manera que se hace más significativa la vivencia de aprendizaje del estudiante en este lenguaje de programación.

La segunda intención de la sistematización estuvo relacionada con el desarrollo de habilidades de pensamiento geométrico y espacial, a partir de la organización lógica de información e ideas que mejoren el análisis y la creatividad en la solución de problemas, en particular, comprender el concepto de algoritmo y utilizarlo para visualizar mejor una situación problema y atender a los procedimientos en Lenguaje Logo para resolverla. A continuación se presentan algunos antecedentes relacionados con el trabajo en el aula, referentes sobre el pensamiento geométrico y espacial, la vivencia en el aula, las consideraciones finales y la bibliografía de soporte.

\section{Antecedentes}

En las investigaciones adelantadas con el uso del lenguaje Logo se distinguen trabajos como el de Guerrero y Chazatar (2001), quienes, a partir de un "Micro cu- rrículo informática Lenguaje Logo", propician un aprendizaje autónomo a través de este lenguaje en medio de una investigación que se constituye como un proyecto de aula multidisciplinario. La propuesta de Candioti (1994), Del clavo al ordenador con Logo, se recogen aspectos significativos relacionados con Logo, el interés radica en que el estudiante conozca y maneje las premisas de Lenguaje Logo y las ubique espacialmente.

La Guía Didáctica del Lenguaje Logo, a diferencia de las anteriores, genera actividades que retan las habilidades del estudiante, recoge un trabajo que ha estado elaborando y experimentando el Grupo LOGO/MADRID. En los años 1987-1988 fue experimentado en 30 centros del país en el marco de un plan de innovación educativa, seleccionado por el Ministerio de Educación (Arias, Bautista, Cano, Domínguez, et al, 1998). En esta propuesta se introducen los conceptos de una forma heurística, de aprendizaje por descubrimiento, y las actividades se realizan en grupo.

En la propuesta "Logo forKids: An introduction", Bob DuCharme (2002), da a conocer esta herramienta de aprendizaje y orienta su propuesta hacia estudiantes de Middle School (básica); observa las primitivas y comandos de esta aplicación, explica cómo se guardan las actividades realizadas, propone tipos de laberintos en los cuales, a partir de una imagen dada al estudiante o grupo de estudiantes, debe desplazar la tortuga sorteando obstáculos. Esta propuesta está orientada a conocer cómo funciona este leguaje de programación, en donde los estudiantes analizan, proponen y explican las soluciones al problema dado.

La experiencia con el lenguaje Logo, a nivel de primaria, en el Colegio Gimnasio Colombo Británico, Bogotá, ha tenido dos momentos: el primero, relacionado con el nivel de exigencia que implica el desarrollo de las actividades propuestas, en la solución de situaciones problema y en la búsqueda y/o creación de sus propias soluciones. El segundo momento, está relacionado con el interés y satisfacción de los estudiantes, dado que en la mayoría de los casos es la primera vez que trabajan con un software que les permite explorar la geometría y la programación. Parte de este trabajo se realiza libremente, los estudiantes pueden crear sus propios diseños teniendo en cuenta ciertas condiciones. 
La institución ya se ha trabajado con lenguaje Logo en los grados primero, segundo y tercero de primaria, específicamente la ubicación espacial, desplazamiento y rotaciones de 90 grados y en la construcción de diseños geométricos libres y otros que han sido propuestos, tales como segmentos de rectas, paralelos y perpendiculares, cuadrados, rectángulos, etc. En grado cuarto se le pide al estudiante realizar construcciones que requieren giros diferentes a $90^{\circ}$, utilizar comandos que repiten instrucciones y análisis que requieren habilidades de cálculo mental, entre otros asuntos. Particularmente, en la presente experiencia, se propusieron situaciones que requerían el uso de los pensamientos geométrico y espacial como elementos importantes en la construcción de procedimientos (programas) en lenguaje Logo, que les permitieran a los estudiantes organizar sus ideas y así solucionar de forma más significativa las situaciones problema propuestas en clase.

Una de las experiencias llevadas al aula con poliminoes, particularmente en la construcción de pentominós, fue realizada por Cárdenas con niños de quinto grado de Básica Primaria en el IED Entre Nubes Sur Oriental. El profesor:

Considera que se hace necesario proponer actividades que contribuyan no sólo a la exploración de las ideas que poseen los niños sobre el espacio, sino al enriquecimiento de las mismas a favor del proceso de construcción y aprehensión espacial, fundamental para su movilización, desplazamiento, ubicación y orientación en el mundo cotidiano [y que] este trabajo permite ubicar la forma como los niños afrontan y se relacionan con la actividad, sus dificultades e inconvenientes; al mismo tiempo, se vislumbra la construcción de la idea de pentominó. La vivencia de la clase posibilita que los niños desarrollen la creación, reflexión, conversación, diseño de dibujos y el cuestionamiento continuo sobre la actividad planteada. (Cárdenas, 2006, pp. 45-48).

Mientras, la profesora Malagón considera, con respecto a la clase, que:

Una clase diferente de matemáticas debe permitir, a partir de una actividad (o problema) motivante, que los muchachos interactúen como grupo, aportando toda su creatividad, realizando razonamientos que les sustenten su trabajo y que aunque no se estén considerando contenidos (programas) de una manera formal, sí se haga "uso" de ellos. (Malagón, 1995, p. 30).

\section{Pensamiento espacial y pensamiento geométrico}

En el mundo actual el elemento visual ha cobrado gran relevancia en los diferentes procesos de aprendizaje, puesto que los medios tecnológicos han permitido un mejor aprovechamiento del mismo; se puede presentar mayor información a los estudiantes con un menor esfuerzo de abstracción, sin embargo, surge una problemática acerca de cómo aprovechar el potencial de esta información, ya que si no se comprende y se significa, entonces se vuelve simplemente un cúmulo de datos sin sentido para los estudiantes y el maestro.

Esta situación lleva a diseñar o pensar en la implementación de actividades que permitan desarrollar habilidades de observación, clasificación, análisis y comprensión, de tal forma que posibiliten la construcción de conocimiento significativo por parte de los estudiantes. Una ruta posible para lograr aprendizajes significativos en el aula, es diseñar propuestas que tengan en cuenta el pensamiento espacial y geométrico, dado que estas dos formas están presentes en la realización de actividades relacionadas con el lenguaje de programación Logo, desde el cual se contribuiría al desarrollo de habilidades en los estudiantes.

En este sentido se distingue que atendiendo al "pensamiento espacial", se desarrollan habilidades de observación que permiten reconocer objetos o elementos y su ubicación, para así poder interactuar con ellos. Vargas (2.000), plantea que en la representación visual se requiere que los datos sean fácilmente convertidos en información y se puedan interpretar "fácilmente". La perspectiva espacial aumenta su interpretabilidad, así, se pueden representar espacialmente datos no espaciales. Por ejemplo, el uso frecuente de pirámides, tartas, barras, cubos, etc.

El pensamiento espacial puede desarrollarse para incrementar las habilidades mentales, su utilización eficaz requiere del enfoque que caracteriza a la educación mental: "saber comprender, integrar y extender el propio aprendizaje. Un aprendizaje intencional, dirigido por un objetivo y controlado conscientemente". En el pensamiento geométrico se logra realizar clasifi- 
caciones a partir de la observación, que permiten la utilización de diferentes tipos de lenguaje (símbolos, palabras, formulas, etc.), también permite establecer relaciones de igualdad, incidencia, perpendicularidad, simetría etc. Van Hiele, citado en Corberan (1989), plantea que en el pensamiento geométrico hay distintos niveles, a saber:

- Reconocimiento: los estudiantes son capaces de reconocer las formas geométricas como una unidad sin características particulares; describen las formas geométricas a partir semejanzas con objetos de su entorno.

- Análisis: los estudiantes identifican partes y propiedades matemáticas de las figuras geométricas; pueden enunciar de manera informal otras propiedades, no pueden hacer clasificaciones lógicas a partir de los elementos o propiedades de las figuras.

- Clasificación: aparece la capacidad de razonamiento formal matemático en los estudiantes; pueden reconocer algunas propiedades, deducir otras y a partir de estas clasificar las figuras en diferentes familias. Al no entender la estructura no perciben la necesidad de encadenarlos, que permitiría entender la estructura axiomática de la familia.

- Deducción Formal: logran hacer razonamientos lógicos formales, demostraciones que incluyen cierto número de pasos que tienen sentido para los estudiantes, mostrando que ya pueden comprender la estructura axiomática de las matemáticas y reconocer que se puede llegar a un mismo resultado desde diferentes premisas.

Visto de esta forma, el pensamiento geométrico no depende del desarrollo biológico del estudiante, sino de la adquisición de los elementos necesarios que le permitan abordar las problemáticas propuestas en los diferentes niveles de escolaridad. Piaget (1986), por su parte, plantea unas etapas en la construcción del espacio y de las propiedades geométricas del espacio. Considera que la percepción del espacio y las diferentes construcciones de nociones, relaciones $y$ propiedades geométricas, evolucionan paralelamente al individuo. El desarrollo geométrico tiene dos etapas: una acumulativa y otra de transición gradual.
Una de estas etapas está relacionada con el "espacio percibido o vivenciado e inicio al espacio representado"; parte de la experiencia inmediata y de la vivencia motriz desde una perspectiva centrada en el sujeto, se afianzan las propiedades topológicas de proximidad, separación vecindad, etc. A continuación se comienza la percepción espacial funcional, más objetiva y menos centrada en el sujeto (de 0 a 5 ó 6 años). En la capacidad de orientación espacial la lateralidad juega un papel fundamental.

La otra etapa está relacionada con el "espacio pensado/ representado/ estructurado", en el que se presenta un incremento del análisis de las propias percepciones y un mejoramiento de las imágenes mentales que permiten una transición de la realidad a un pensamiento geométrico más evolucionado, que se caracteriza por la comprensión y dominio de propiedades matemáticas del espacio, como las proyectivas y euclídeas, orientaciones, volúmenes, perspectivas, escalas coordenadas, etc. Esto continúa y se perfecciona a lo largo de la vida. Se presenta una relación inversa entre la aparición histórica de las propiedades geométricas y su asimilación por parte del sujeto:

- Propiedades Topológicas: son propiedades y relaciones cualitativas elementales: vecindad, separación, sucesión espacial, envolvimiento, etc.

- Propiedades Proyectivas: distancia, forma y tamaño del objeto, también nociones de abajo, arriba, adelante, atrás, derecha, izquierda, etc. Su formación requiere de procesos complejos de representación mental (7 a 8 años de edad).

- Propiedades Euclídeas: se refieren a situar objetos con respecto a un sistema de referencia, implicando la necesidad de medidas como la longitud, superficie, ángulos volúmenes, etc. (6 años o más).

Así, aparece una diferencia entre Piaget y Van Hiele, ya que para el primero el aprendizaje matemático y el desarrollo intelectual están íntimamente ligados al desarrollo biológico, mientras que Van Hiele es más explícito cuando afirma que la imposibilidad de los niños para pensar lógicamente no procede de la falta de maduración, sino de una ignorancia de las reglas de la lógica. El niño no tiene a su disposición las estructuras a partir de las cuales se originan las preguntas. 
Desde el Laboratorio Latinoamericano de Evaluación de la Calidad de la Educación (LLECE), se define el pensamiento geométrico como una forma de pensamiento matemático; sin embargo, no es exclusiva de él, este supone el conocimiento de un modelo del espacio físico tridimensional. Se considera que a partir del pensamiento geométrico se pueden desarrollar tres capacidades en los estudiantes:

- Vista espacial: si el alumno no consigue ver la realidad de lo que dibuja, el aprendizaje de cada uno de los lenguajes se convertirá en una suerte de intrincado jeroglífico, Ileno de trucos y trampas en las que siempre se acabará cayendo por no recordar esta o aquella otra propiedad o dinámica operativa (Geometría Descriptiva).

- Representación espacial: el hecho de adquirir conocimientos del espacio real a través de la intuición geométrica es lo que se llama la percepción espacial. La percepción espacial se ocupa de obtener un mensaje por medio de la "lectura comprensiva" de las formas y relaciones espaciales de nuestro entorno: reconocimiento de formas, propiedades geométricas, transformaciones y relaciones espaciales. (La Geometría puede ser caracterizada como el estudio de las experiencias espaciales).

- Imaginación espacial: esta depende de cada persona y es utilizada de distinta manera de acuerdo a las experiencias y el acervo cultural. El comparar las distintas formas de pensar a partir de una situación descriptiva particular permitiría entender las diferentes formas de pensar.

\section{Vivencia en el aula}

En la actividad se utiliza el Lenguaje Logo en la solución de situaciones problema que impliquen el pensamiento geométrico y espacial para identificar elementos comunes y así crear algoritmos. Se considera que el Lenguaje Logo es un lenguaje de programación; un lenguaje de computadora, un lenguaje para aprender, una herramienta útil para enseñar el proceso de aprendizaje y de pensamiento, en especial el pensamiento geométrico y el espacial. Esta experiencia se distancia de ideas que consideran que en la programación con computadores es común que se manejen lenguajes extensos, técnicos y confusos que los estudiantes tienen que aprendérselos de memoria.
La actividad se realiza con estudiantes de cuarto grado de Básica Primaria del Colegio Gimnasio Colombo Británico, Bogotá; tiene como intención desarrollar habilidades de pensamiento geométrico y espacial, a partir de la organización lógica de información e ideas que mejoren el análisis y creatividad en la solución de problemas a través del trabajo con Lenguaje Logo.

La metodología es activa, se busca que el estudiante participe en sus procesos de aprendizaje, él es quien propone las soluciones a los problemas inicialmente planteados por el profesor, logrando así mejorar su nivel de autonomía y responsabilidad. Para recoger la información de la vivencia en el aula se tuvieron en cuenta los cuadernos de los estudiantes, las hojas de trabajo en grupo, fotos y las construcciones en el ordenador. La actividad se realizó en cuatro momentos: los dos primeros se realizaron sobre papel y los dos últimos fueron llevados al ordenador con el uso del graficador Paint y del Lenguaje Logo.

\section{Primer momento: imaginando, analizando y dibujando en el papel}

En esta etapa se buscó identificar los elementos del pensamiento geométrico y espacial que emergen cuando se trabaja con una hoja de papel, lápiz y regla. Se indaga por las estrategias que utilizan los estudiantes para dibujar en el papel e identificar las relaciones que establecen cuando realizan el dibujo.

La actividad partió del ejercicio de dibujar un cuadrado con un perímetro de $28 \mathrm{~cm}$ en una hoja; se solicitó a los estudiantes que lo hicieran utilizando regla y lápiz y comentaran cómo hicieron dicha construcción. Posteriormente se pidió que dibujaran un cuadrado de $52 \mathrm{~cm}$ de perímetro, ilustrado en una hoja de papel cuadriculada, a continuación, debieron describir los pasos tenidos en cuenta para realizarlo y mencionar las semejanzas o diferencias con el ejercicio anterior. Finalmente, se les propuso que dibujaran tres o más cuadriláteros de $16 \mathrm{~cm}$ de perímetro. La intención de variar el perímetro fue la de distinguir las estrategias, relaciones y rasgos que permiten identificar características del pensamiento geométrico y espacial. En la foto 1 se observa uno de los trabajos. 


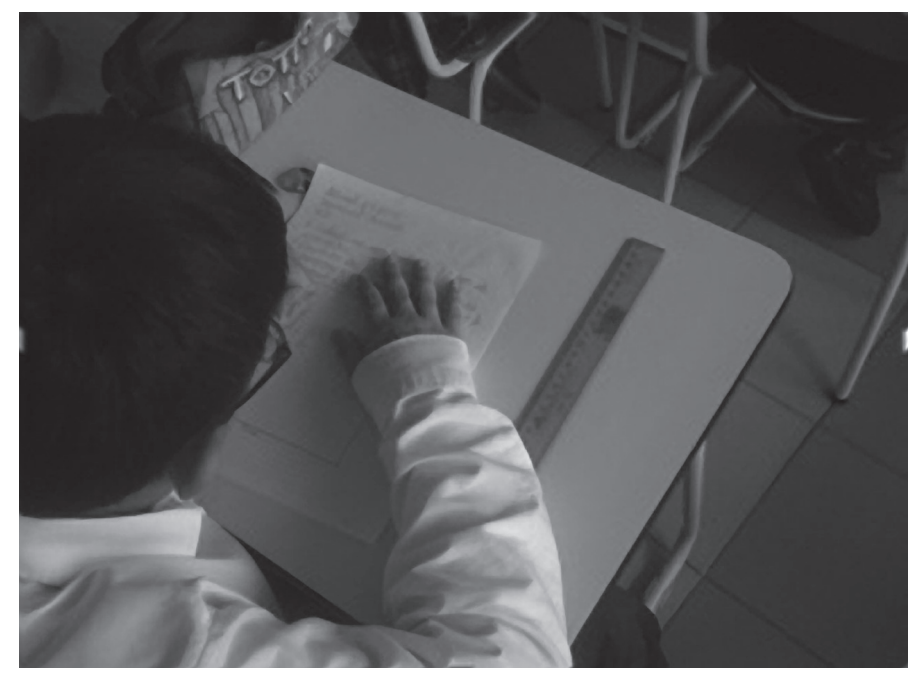

Foto 1. Construcción del cuadrado en papel

En cuanto a las construcciones sobre cómo dibujar un cuadrado de $28 \mathrm{~cm}$ de perímetro en una hoja, se plantearon algunas opciones, como:

Divido y multiplico 28/7, igual 4. Entonces $7 \times 4=28$.

1) Divide 28/4=27 2) El 7 es $7 \mathrm{~cm}$, los cuales tienes que tener en cada lado. 3) Realiza con la regla $7 \mathrm{~cm}$ en cada lado.

$7+7+7+7=28$. 2) Suma 7 cuatro veces. 3) Ese el cuadrado de 7 centímetros.

Yo dividí 28 en 4 y supe la medida que necesitaba usar para cada lado que era 7, y le hice el cuadrado de $28 \mathrm{~cm}$ de perímetro.

La gran mayoría de los estudiantes reconocieron la forma del cuadrado y la dibujaron, se presentaron algunos casos donde se plasmaron rectángulos en lugar de cuadrados; al parecer esto se debió a la manera como se repartió el perímetro; la mitad del grupo entiende el concepto de perímetro y cómo aplicarlo en un cuadrado. En los dibujos realizados, la figura propuesta es identificada manteniendo los ángulos iguales, lados del mismo tamaño y el perímetro dado, a partir de esto se puede pensar que los estudiantes reconocen la figura y su número de lados. Algunas de las figuras se muestran en la Tabla 1.

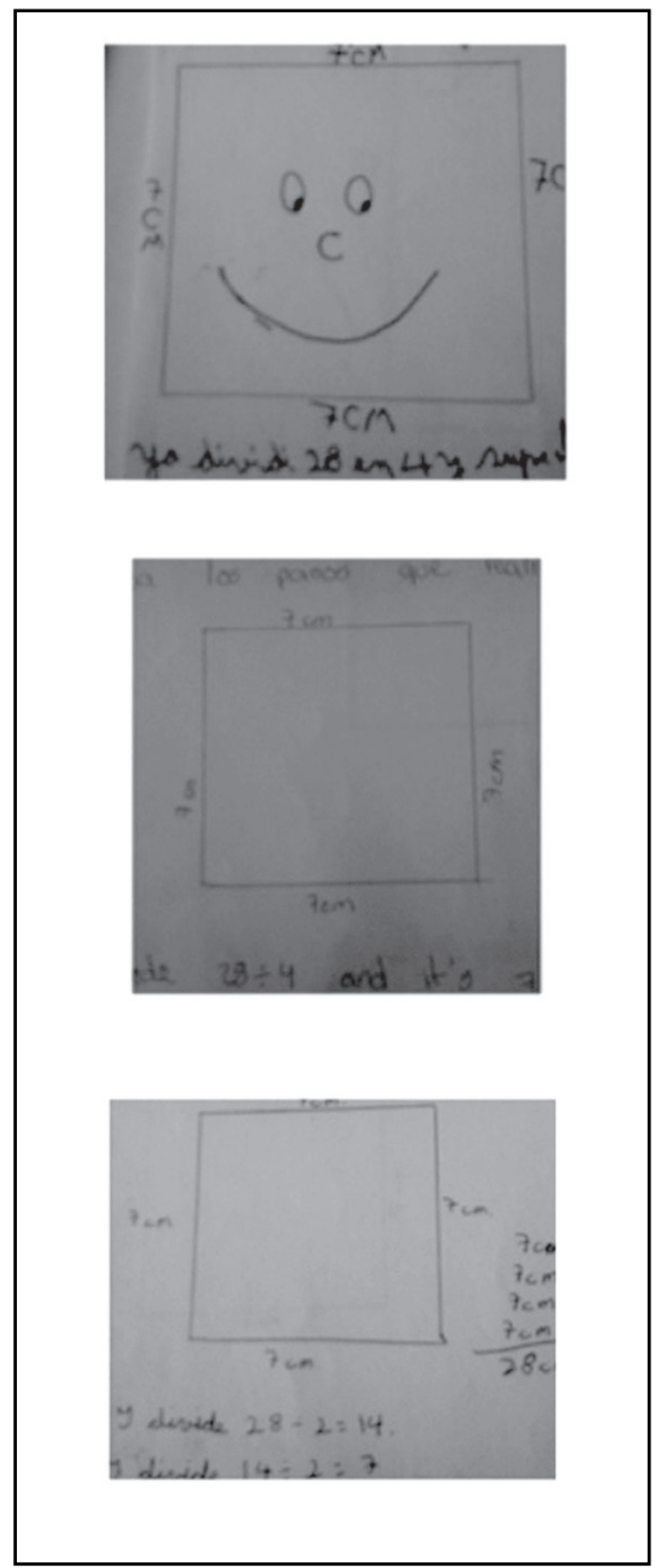

Tabla 1. Cuadrados de $28 \mathrm{~cm}$.

La mayoría de estudiantes hizo análisis e identificó ciertas propiedades geométricas de la figura del cuadrado, ya que a partir de la relación de igualdad de los lados fueron capaces de establecer operaciones de división implícita o explícita para obtener el valor de cada lado a partir del perímetro dado.

En cuanto a la solicitud de cómo dibujar un cuadrado de $52 \mathrm{~cm}$ de perímetro en una hoja de papel cuadriculado, y que luego se ilustren tres o más cuadriláteros con $16 \mathrm{~cm}$ de perímetro, se tienen algunas construcciones como las que se presentan en la Tabla 2. 


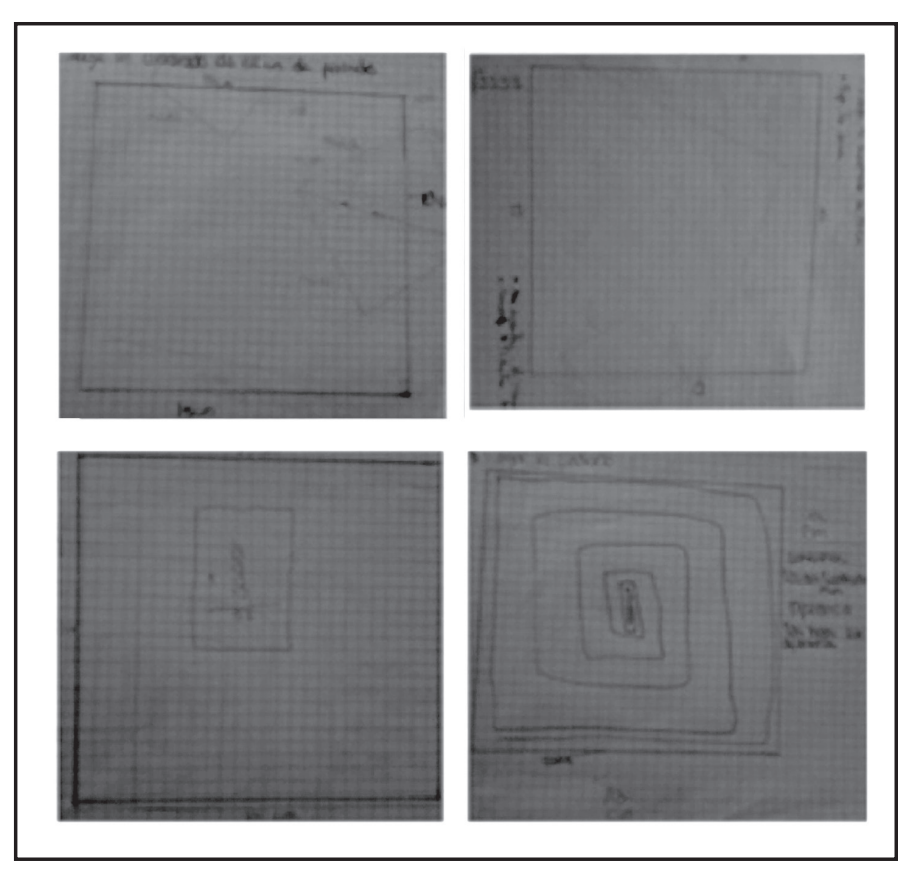

Tabla 2. Cuadrado de $52 \mathrm{~cm}$.

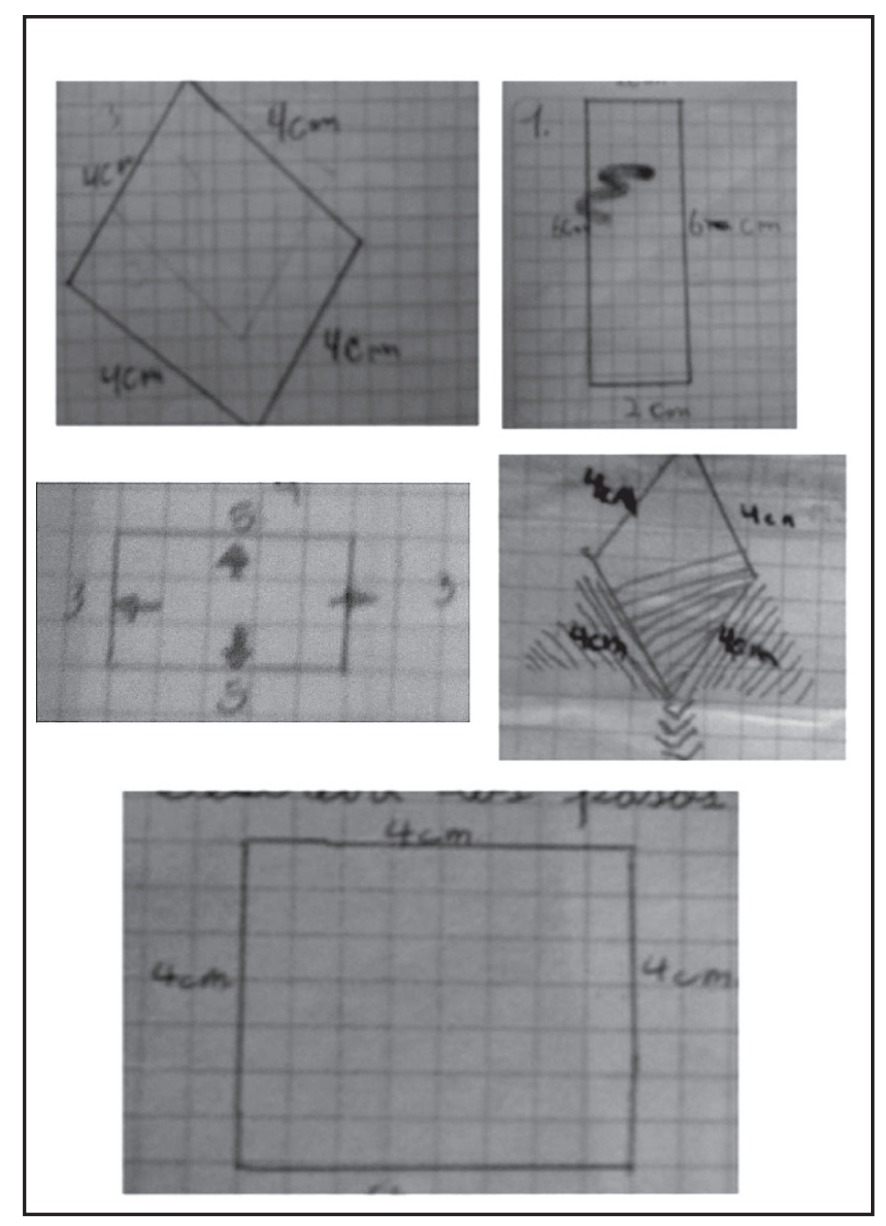

Tabla 3. Cuadriláteros construidos
A diferencia de las construcciones anteriores, en esta, son los estudiantes quienes proponen las figuras a partir de las condiciones dadas (número de lados y perímetro). Se pudo distinguir, no sólo el conocimiento de las figuras, sino su agrupación por el número de lados, ya que se realizaron clasificaciones y el reconocimiento de la figura cuadrado. Un gran número de estudiantes identificó ciertas propiedades geométricas y relaciones matemáticas de las figuras propuestas, lo cual se evidencia cuando expresan que "cuadrado nuevamente pero de diferente perímetro y tres cuadriláteros que tuvieran el mismo perímetro". A partir de la relación entre los sus lados, establecieron operaciones de división implícita o explícita, sumas o multiplicaciones que les permitieran utilizar diferentes valores y aún así obtener el mismo perímetro.

En la construcción en una hoja cuadriculada del cuadrado de $52 \mathrm{~cm}$ de perímetro, los estudiantes afirmaron que:

Primero sumé diferentes números cuatro veces para conseguir el número y encontré que, en las dos actividades, el número del perímetro es par y que en la segunda actividad el cuadrado es más grande que el primero $13+13+13+13=52$. [...] Dividí 52 entre 4 y medio, igual 13; así que cada lado tiene que medir $13 \mathrm{~cm}$ y entonces el dibujo fue el que me facilitó, porque la hoja es cuadriculada y me tocó hacer el mínimo esfuerzo.

En cuanto a la construcción de tres cuadriláteros de 16 $\mathrm{cm}$ de perímetro, casi la totalidad de los estudiantes identificó el concepto de cuadrilátero y las posibles figuras que pueden surgir de este, como rectángulos, rombos, cuadrados, etc.; analizaron y resolvieron la construcción utilizando diferentes métodos para llegar a la respuesta (multiplicación, división, suma y medición directa), establecieron relaciones de igualdad entre pares de lados para encontrar los lados de los cuadriláteros.

\section{Segundo momento: jugando con Poliminoes}

Este momento buscó identificar las formas de operar de los estudiantes al dibujar los poliminoes y describir cuáles son las estrategias, las relaciones y los patrones que siguen de su construcción; en particular, la capacidad de distinguir invariantes en las figuras y reconocer las posibilidades de la construcción de las mismas. El 
ejercicio se adelantó a partir del juego de los "poliminoes" atendiendo a unas condiciones dadas y al patrón inicial de la figura (cuadrado) para construir nuevos poliminoes.

En primer lugar, se caracterizó a los poliminoes como figuras geométricas construidas a partir de cuadrados; en segundo lugar, se definió colectivamente unas condiciones, como que en la construcción desde el segundo poliminoe, se tuviera en cuenta que un lado del cuadrado hiciera parte de otro cuadrado (compartir un lado) y que una figura no fuera la rotación de otra. En tercer lugar, se propuso encontrar el mayor número de combinaciones posibles para luego socializar en el grupo las construcciones que se hicieron a partir del monominoe y, de ahí, los posibles dominoes, triminoes y tetraminoes. Algunas de las figuras obtenidas se observan en la Tabla 4.

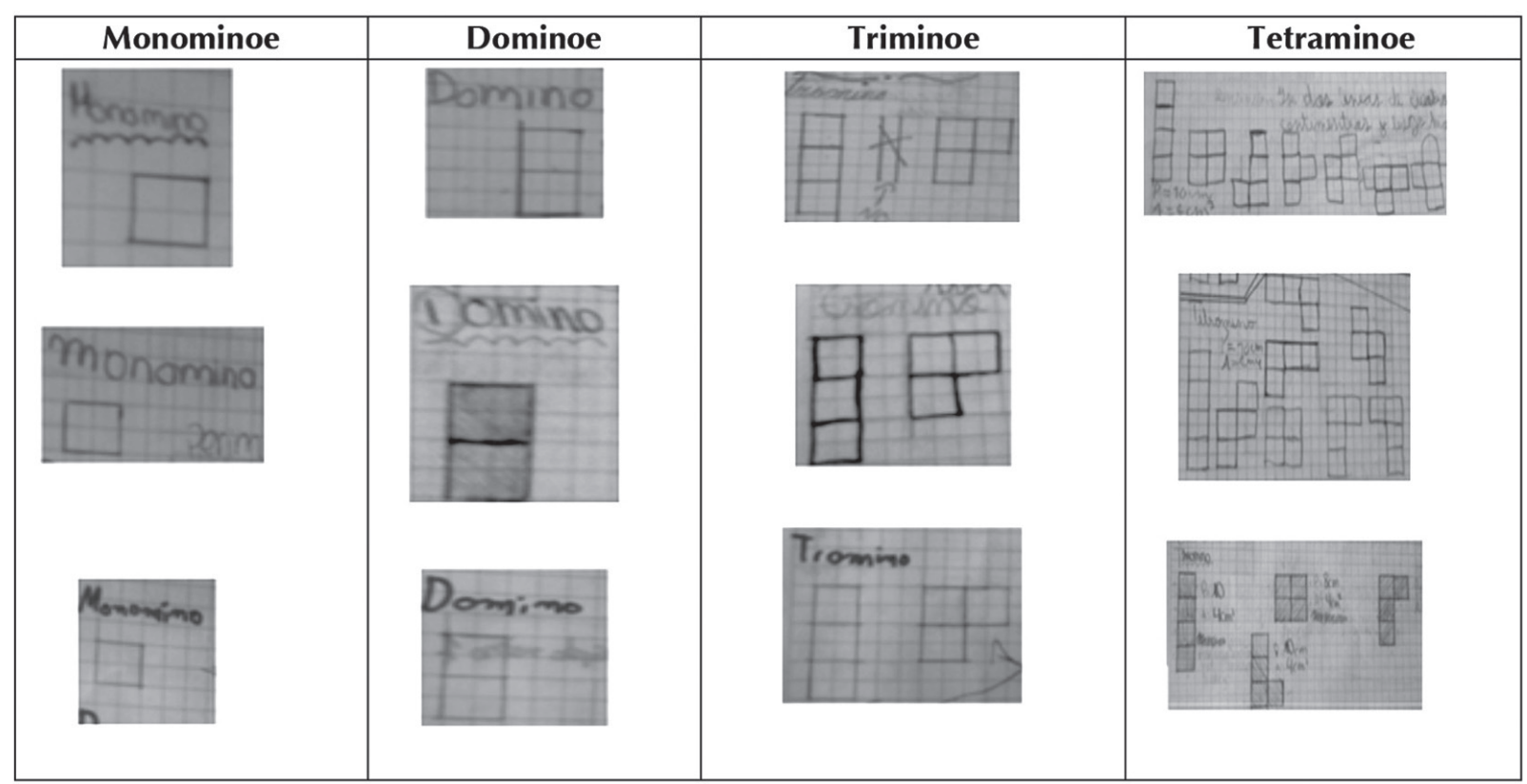

Tabla 4. Poliminoes construidos en clase

Los estudiantes propusieron las figuras a partir de las condiciones dadas; en sus ejercicios se puede distinguir no sólo el conocimiento de las posibles construcciones de las figuras, sino su agrupación por el mismo número de cuadrados que las componen. En la Foto 2 se registra el trabajo de uno de los estudiantes, mientras que, algunos de los comentarios en este trabajo fueron:

Yo moví los cuadrados arriba y en el centro para obtener diferentes figuras, moví los cuadrados para obtener una figura diferente: Poliminoes son cuadrados que van aumentando el perímetro, es importante para que sepamos.

Tetromino: Primero hice 4 cuadrados hacia abajo y así entonces los hice. Hice un cuadrado, Pentomino.

Yo la hice haciendo 4 cuadrados hacia abajo y 1 a la izquierda. Creo que la parte más difícil

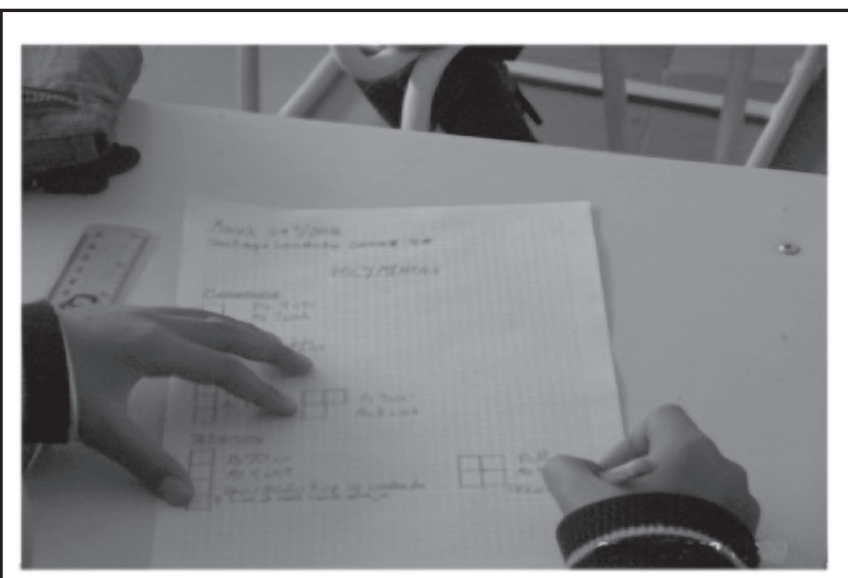

Foto 2. Construcción de uno de los estudiantes era el Tetromino. 
En los dibujos, los estudiantes identificaron y aplicaron las condiciones dadas: que sean compuestos solo por cuadrados del mismo tamaño, que sólo se puedan unir totalmente y no parcialmente por sus lados, hallar el mayor número de combinaciones posibles y que unos no pueden ser el resultado de la rotación de otros. Los niños clasificaron sus construcciones y agruparon las figuras en familias de acuerdo al número de cuadrados que las componen.

Tercer momento: dibujando y creando poliminoes en el graficador Paint

En este momento se buscó comprender las características de los poliminoes; identificar las estrategias que se utilizan al diseñarlos en un programa de diseño gráfico como Paint e indagar sobre los conceptos de área y perímetro, además de las regularidades que emergen en la construcción de las figuras. Los estudiantes construyeron las figuras en el graficador Paint utilizando las opciones de copiar y pegar, calcularon el área y el perímetro de cada una de las figuras para hallar similitudes y diferencias que les permitan hacer nuevas clasificaciones. En la Tabla 5, se presentan algunos poliminoes construidos por los niños.
Para el ejercicio, los estudiantes afirmaron que:

Los Poliminos son cuadrados que se unen para armar una figura, tienen que estar unidos de un lado o más, si no están unidos de un lado completo no es un polimino.

Tromino: los dos trominos tienen la misma área, los dos trominos tienen el mismo perímetro, tienen la misma área porque tienen la misma cantidad de cuadrados A; también tienen la misma cantidad de perímetro porque las dos figuras sólo tienen unido un sólo lado P: 8.

Tetromino: todos los tetrominos tienen la misma área porque tienen cuatro cuadros. A: 4 , seis tetrominos tienen la misma cantidad del perímetro, un tetromino no tiene el mismo perímetro porque está unido de 2 partes.

Las características de un polimino es que todos ellos están hechos a base de cuadrados del mismo tamaño, y por lo menos uno de los lados del cuadrado tiene que estar pegado con otro lado del otro cuadrado (completamente). Dos figuras en Tromino tienen la misma Área: $\mathrm{A}=3 \mathrm{~cm}^{2}$, porque los dos están hechos de tres cuadrados y también tienen el mismo perímetro: $\mathrm{P}=8 \mathrm{~cm}$, porque tienen el mismo número de líneas por fuera. Las siete figuras tienen la misma Área: $\mathrm{A}=4 \mathrm{~cm}^{2}$, porque los 7 son hechos de cuatro cuadrados y seis cuadrados tienen el mismo perímetro: $\mathrm{P}=10 \mathrm{~cm}$, porque tienen el mismo número de líneas por fuera.

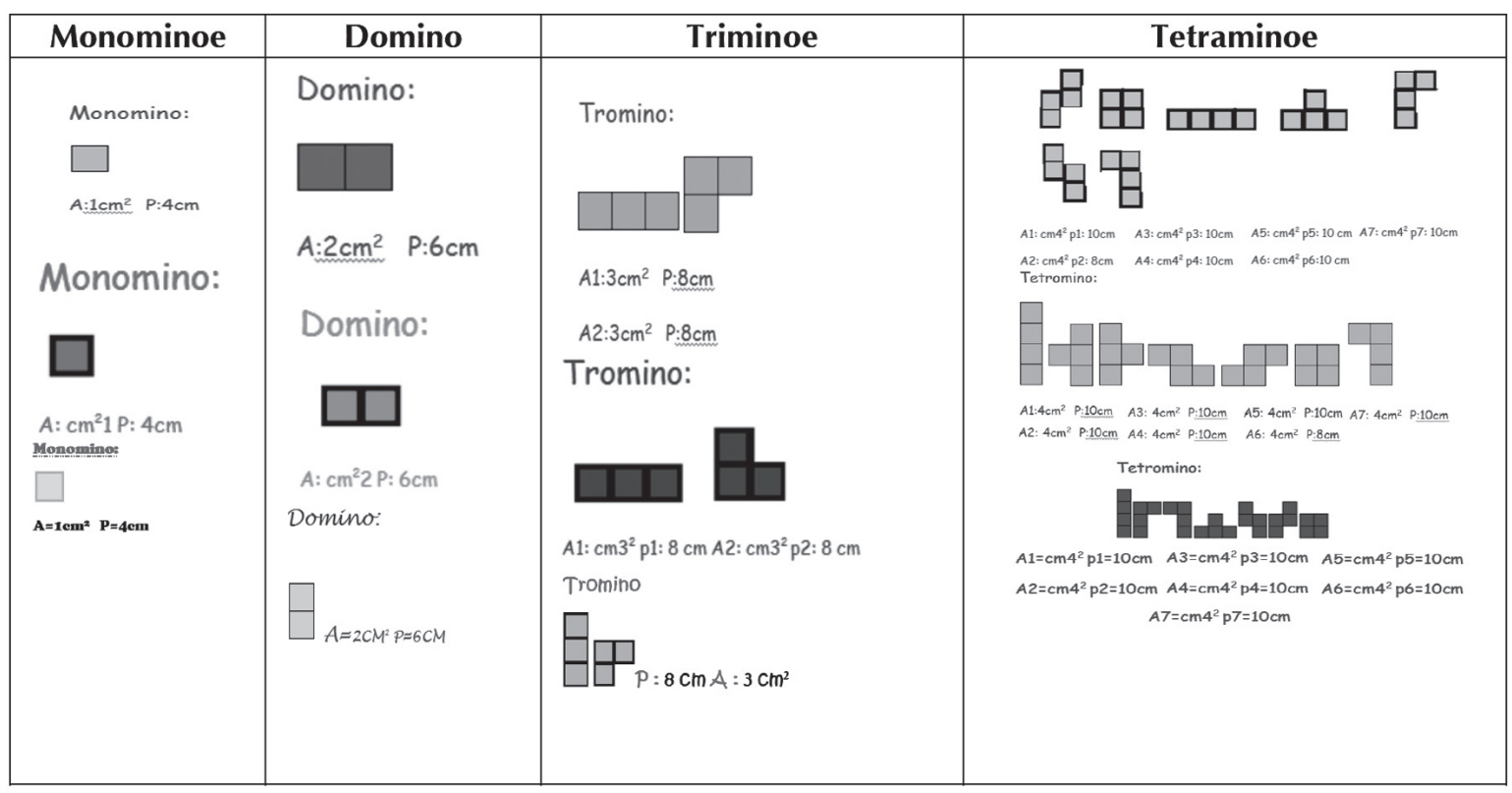

Tabla 5. Poliminoes construidos por los estudiantes 
Los estudiantes reconocen, para este momento, el área de los Poliminoes como la suma del número de cuadrados que los componen, y esto les permite agruparlos y clasificarlos; la mayoría afirma que el perímetro depende del área (número de cuadrados) y que en la mayor parte de los casos la forma no lo afecta, pero algunos llegaron a identificar que la disposición de los cuadrados sí puede afectar el perímetro, reconociendo que entre más lados compartan los cuadrados dentro de una figura, será menor el valor del perímetro, pues serán menos los lados quedan expuestos.

Una vez realizadas las construcciones, los estudiantes ya tienen en cuenta que los poliminoes se conforman de cuadrados, los cuales como condición deben estar unidos completamente por sus lados; además, uno no puede ser la rotación de otro, pero sí se pueden realizar imágenes de estos (invertirlos) y su condición inicial es tener uno más cuadrados; además, son capaces de agruparlos en diferentes familias de acuerdo con los números de cuadrados que los componen, es decir Monominoes, Dominoes, Triminoes, Tetrominoes, Pentominoes, etc. En la Foto 3, se presentan los diseños realizados en Paint.

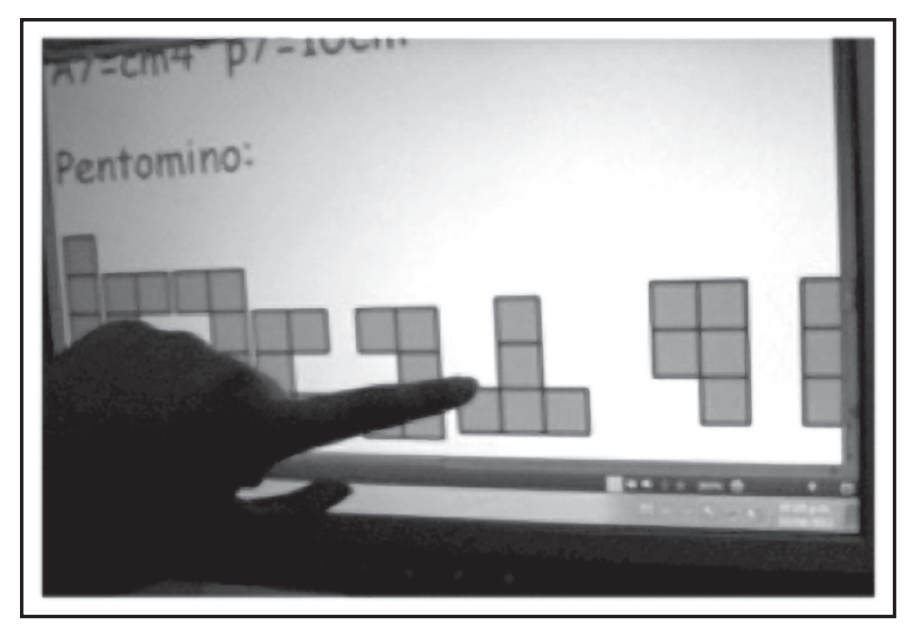

Foto 3. Diseños de poliminoes

Sobre el área y el perímetro los estudiantes expresaron que: "El área es lo que cubre la figura y el perímetro es su contorno, el área es lo que está encerrado en ella y el perímetro son sus bordes, el área es lo que se puede medir en centímetros cuadrados y el perímetro en centímetros solos". Los estudiantes también dijeron que: "cada familia de Poliminoes se caracteriza porque tienen la misma área, aun si las figuras tienen diferente forma, ésta se mantiene, es decir, el mismo número de cuadrados".
En cuanto a los perímetros, en la mayoría de casos los estudiantes afirmaron, como para el área, que estos no varían por la forma pero que, sin embargo, hay momentos en donde sí sucede, obteniéndose un perímetro diferente; esto fue asociado con la disposición de las cuadrados en la figura, es decir, si el número de uniones de estos es mayor en "la parte interna" de la figura; de esta manera (implícita), los estudiantes relacionaron el perímetro de la figura con el número de lados de los cuadrados expuestos externamente. Es por ello que se presentaron afirmaciones como:

Fue interesante y retó mi creatividad a la hora de encontrar las posibles combinaciones que formaban las figuras siguiendo las condiciones dadas inicialmente para su construcción, por otro lado, fue una buena manera de medir el área y el perímetro de una figura.

Para finalizar, se generó un trabajo en el ordenador utilizando Paint, un programa de diseño grafico, y un procesador de texto, Microsoft Word, ejercicio en el cual se pudo distinguir que, conociendo las figuras, los niños son capaces de construirlas y de establecer relaciones a partir del perímetro y del área; logran más combinaciones en el ordenador que en el papel, dado que su experiencia con esta herramienta en la clase les resulta familiar; conocen y manipulan las herramientas de los programas; al tiempo que identifican las formas de operar al dibujar los poliminoes y describir estrategias, relaciones y patrones que siguen al construirlos, al distinguir invariantes en las figuras y reconocer las posibilidades de la construcción de las mismas.

El desarrollo de la actividad permitió hasta este momento evidenciar que para el trabajo en papel, algunos de estudiantes tuvieron dificultades en el trazo y la utilización de la regla, mientras que en las actividades realizadas con el graficador Paint, los problemas surgieron gracias al desconocimiento de algunas herramientas ofrecidas por el ordenador. Se presentaron confusiones a la hora de dibujar los cuadriláteros con perímetro específico, ya que sólo tuvieron en cuenta las medidas de dos de los lados con respecto al perímetro dado (las figuras fueron el doble del tamaño solicitado). En cuanto a la construcción de Poliminoes se presentaron al principio algunas confusiones debidas a que los estudiantes creían que la rotación no válida era la de $90^{\circ}$ y los rotaban hasta los $180^{\circ}$. 
Se distingue además que la mayoría de los estudiantes comprende los conceptos de rotación, área y perímetro de una figura plana y cómo medirlos. La actividad les ha permitido desarrollar habilidades tanto de pensamiento geométrico (topológicas, proyectivas y euclídeas), como de pensamiento espacial (interactuar con elementos que conforman una figura para obtener otra), al realizar la construcción de los diferentes poliminoes. En el pensamiento geométrico se logró clasificar, analizar y reconocer aspectos de las figuras.

\section{Cuarto momento: dibujando, creando y recreando poliminoes en el ordenador}

Este momento consta de dos partes, una en la que se busca que el estudiante comprenda y maneje el lenguaje programación Logo, y otra en la que identifique los elementos del pensar geométrico y espacial. Se propuso que el estudiante asumiera el reto de dibujar el cuadrado en el computador, que diera significado a cada una de las instrucciones y algoritmos en este programa y, a partir de las construcciones, tradujera sus representaciones en un lenguaje de programación Logo, empleando los comandos de avanzar, rotar y repetir. Se propuso la construcción de algunos cuadriláteros de diferentes tamaños y la identificación de la posición correcta para dibujar los diferentes cuadrados que componen un Poliminoe y sus representaciones. Para finalmente, a partir de la utilización del comando repeat, graficar cuadrados que conformen los diferentes poliminoes en Lenguaje Logo.

En este trabajo los estudiantes crearon programas informáticos Ilamados "Procedimientos" en lenguaje Logo, los cuales graficaron, a partir de comandos, los diferentes poliminoes. Los estudiantes demostraron un manejo espacial que incluye posición, rotaciones y traslaciones. En la Tabla 6, se presentan algunos de los procedimientos realizados por los estudiantes.

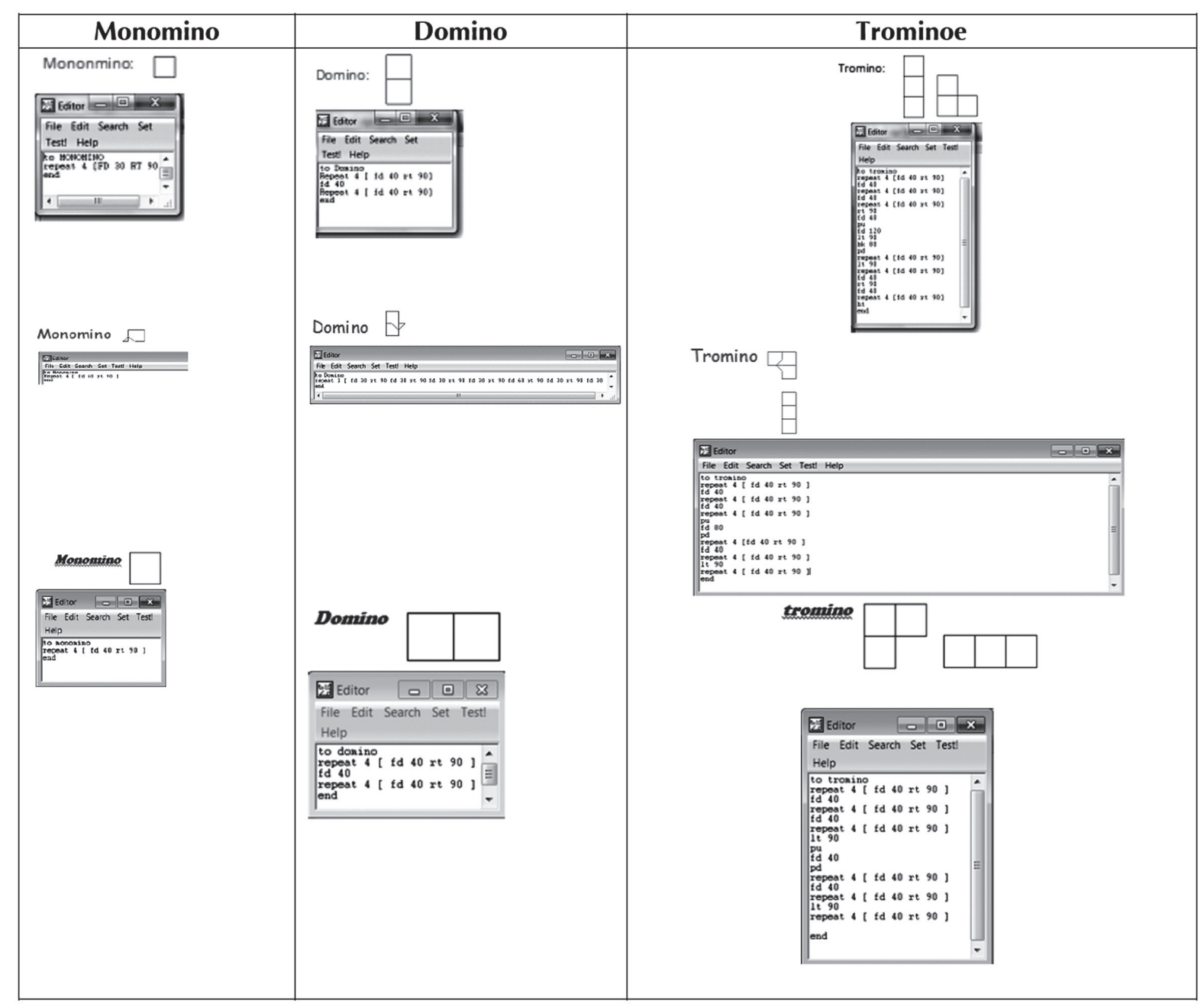

Tabla 6. Procedimientos realizados por los estudiantes 
En el ejercicio de construcción, los estudiantes crearon sus poliminoes (monominoe, dominoe y trominoe) a partir de los comandos de lenguaje logo que ya conocían gracias a sus experiencias de años anteriores (avanzar, retroceder, rotar y repetir); de esta manera, fueron capaces de realizar programas Ilamados "procedimientos", que guardan y ejecutan los comandos utilizados y elaboran un documento de la vivencia de este trabajo. Algunos de los comentarios al respecto fueron:

Primero hay que saber qué significa cada comando, como FD, que significa adelante, RT, significa a la derecha y LT a la izquierda; REPEAT significa repetir cualquiera de los comandos y PU es no hacer líneas; PD significa hacer líneas: como levantar y bajar. Yo encontré la figura uniendo los comandos y sacando un sólo resultado para guardarlo y unirlo con su nombre, en este caso Tromino.

Primero tenemos que saber cuál es la figura, en este caso tromino; también hay que conocer bien los comandos: FD significa adelante, RT significa vuelta a la derecha, LT significa vuelta a la izquierda; REPEAT significa repetir cualquier comando que selecciones, PU significa que puedes mover la tortuga sin que escriba, PD significa que después de usar el PU, la tortuga vuelve a escribir. Yo logré crear la figura uniendo varios comandos con varias combinaciones en REPEAT.

Para hacer un programa para hacer un "Tromino", tuve que usar los comandos "Forward, Backward, RightTurn, LeftTurn, Pen Up etc.", del programa Microsoft Windows Logo, lo cual fue complejo porque tenía que hacer un comando y ver si estaba bien, y usaba un comando para no tener que escribir 4 veces lo mismo, llamado "Repeat"; con ese comando se repite cuantas veces quiera, nada más tenía que copiar ese comando y poner otros para tener un tromino.

En la Foto 4, se registra a un estudiante realizando los procedimientos en Lenguaje Logo.

Foto 4. Trabajo con el Lenguaje Logo

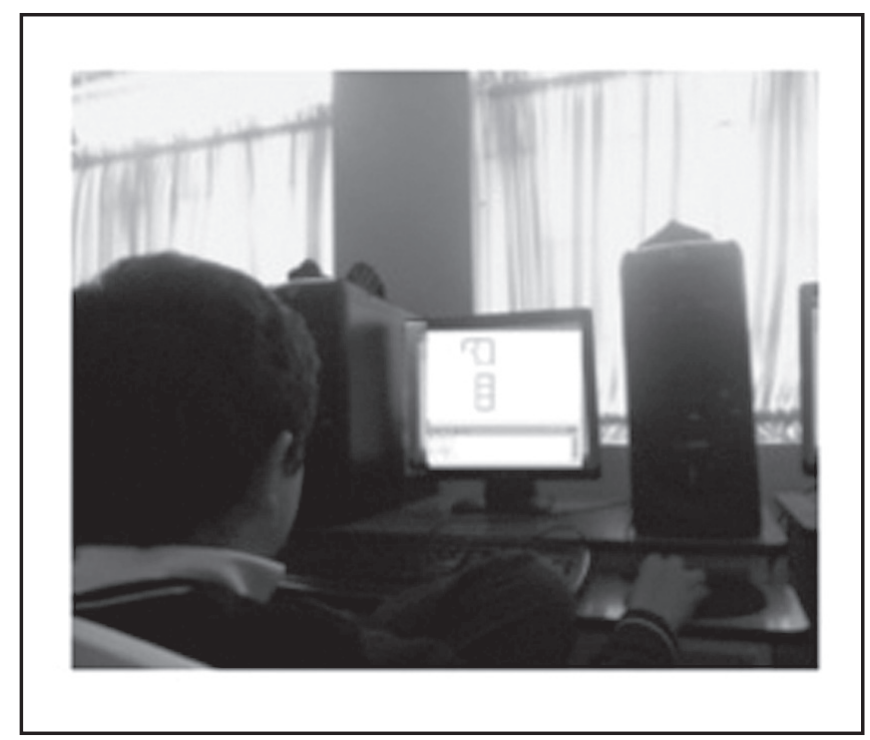

La mayoría de los estudiantes identificaron la simetría del cuadrado y la utilizaron en forma de los comandos: avanzar, girar un ángulo recto y repetir este procedimiento cuatro veces, obteniendo así el cuadrado. A partir de las construcciones de los estudiantes en Logo, se hizo posible hacer evidente un manejo de la lateralidad y del espacio, ya que eran capaces de ubicar correctamente la tortuga (coordenadas), orientarla (dirección) y a desplazarla adecuadamente (traslación), de acuerdo con la figura que deseaban realizar.

\section{Comentarios finales}

La vivencia en el aula fue significativa para los estudiantes y el profesor, generó interés, retos y conciencia de realización al construir sus poliminoes manipulando papel, lápiz y regla; dibujando en el programa Paint y utilizando el Lenguaje Logo.

Se hizo evidente un aumento del nivel de los estudiantes para la construcción de los procedimientos que grafican los poliminoes a través del uso de lenguaje Logo, que les ha permitido integrar la parte espacial con los comandos de un lenguaje de programación, además de las habilidades para ubicarse espacialmente en el plano virtual del software educativo, y realizar rotaciones y traslaciones necesarias para recrear lo poliminoes en esta aplicación.

La actividad en el aula enriqueció las habilidades de pensamiento espacial y geométrico de los estudiantes 
de grado cuarto de Básica Primaria del Colegio Gimnasio Colombo Británico.

La construcción de poliminoes posibilitó un avance en cuanto al pensamiento geométrico, realizar clasificaciones, análisis y reconocimiento de las características de las figuras, el perímetro y el área. Estos aspectos se presentan como hechos implícitos en cada uno de los trabajos realizados en el papel, en los programas Paint

Arias, J., Bautista, M., Cano, J., y Dominguez, B. (1988). Guía didáctica del lenguaje Logo. Madrid: Grupo Logo.

Candioti, C., y Martín, J. (1994). Del clavo al Ordenador. Programas de Nuevas Tecnologías de la Información y de la Comunicación. Madrid: Secretaría General de Educación y Formación Profesionales.

Cárdenas, O., Pedreros, R., Becerra, J., et al. (2006). Ambientes de aprendizaje en el aula. Una experiencia en Colectivo. Bogotá: Alcaldía Mayor de Bogotá, IDEP.

Corberan, R. (1989). Didáctica de la geometría modelo Van Hiele. Madrid: Servei de Publication Universitat de Vanencia.

Ducharme, B. (2002). Logo forKids: An Introduction. New York: NCTM.

Guerrero, E., y Chazatar, A. (2011). Aprendizaje autónomo a través del Lenguaje Logo. Proyecto de aula multidisciplinario mediante la utilización de las TICS. Pasto. y en Lenguaje Logo. En cuanto al pensamiento espacial, los estudiantes fortalecieron y enriquecieron sus habilidades de observación, que fue la que les posibilitó ubicar los cuadrados de distintas formas para crear las diferentes combinaciones de los poliminoes.

\section{Referencias}

Mlagón, Y. (1995). Una opción de clase de matemáticas a nivel medio. Revista Nodos y Nudos. Universidad Pedagógica Nacional, 1 (1), 30-33.

Piajet, J. (2009). La Psicología de la inteligencia. Barcelona: Book Print.

Vargas, P. (2000). El pensamiento espacial es parte de todas las formas de conocimiento, en lo Microcósmico y en lo Macrocósmico. Buenos. Aires: Escuela Mentat.

\section{Diálogo del conocimiento}


Son varias las sorpresas que nos encontramos al leer este trabajo. Indudablemente nos muestra la posibilidad de vincular de una manera tan significativa, que se vuelve "natural", un conjunto de actividades que son, por una parte deseables y, por otra, exigentes de atención, cuidado y dedicación.

Para el autor, preocupado de las exigencias curriculares, el mérito fundamental de la actividad que nos plantea está en la posibilidad de articular coherentemente las previsiones curriculares del pensamiento geométrico con la utilización de un programa para dibujar y la iniciación en la programación, en un ambiente de clase alegre, participativo y colaborativo.

Pero indudablemente cuando se ha leído el artículo, lo que encontramos es que existen otras razones para ver esta actividad como un ejemplo excelente del vínculo que se puede lograr entre el interés por lograr las metas y la voluntad para superar las dificultades que se presentan. Este vínculo que se concreta en la alegría que acompaña el trabajo en clase con las satisfacciones por las conquistas que se logran está mediado al parecer por la estructura de la actividad en términos de retos. Los retos para los niños se constituyen en un acicate permanente que dinamiza sus inquietudes y deseos.

Yendo un poco más lejos, si bien los tres elementos que orientan la actividad, son importantes, existen otras consideraciones no menos interesantes, que debemos señalar.

En primer lugar, el desarrollo de las clases permite que se de un ambiente en el que el protagonismo y la libertad intelectuales se constituyen en las claves: las definiciones de área y perímetro, por ejemplo, son construidas mediante recurrencias inventadas, de tal suerte que las propiedades no son características de los objetos sino emergencias en las interacciones y las definiciones surgen entonces de las actividades y de las necesidades que aparecen cuando el hacer en el aula se desarrolla buscando metas, sin la existencia de caminos predeterminados para conseguirlas.

De esto se podría continuar hablando, de las regularidades, que son elaboraciones de los estudiantes, del protagonismo de los grupos al ir elaborando novedades que surgen en la acción y, finalmente, de la acción como línea conductora.

Cómo sería de interesante proyectar algunas de estas actividades a situaciones con niños más grandes antes de que se den esas pérdidas que tanto se han denunciado, las pérdidas de la curiosidad y la creatividad.

Dino Segura 